\title{
A METHOD TO DETERMINE THE INTRINSIC AXIAL RATIOS OF INDIVIDUAL TRIAXIAL GALAXIES
}

\author{
Ortwin E. GERHARD ${ }^{1}$ and Mario VIETRI ${ }^{2}$ \\ ${ }^{1}$ Max-Planck-Institut für Astrophysik, Garching, West Germany \\ ${ }^{2}$ Osservatorio Astrofisico, Firenze, Italy
}

ABSTRACT. We present a method, partly geometrical and partly dynamical, to determine the intrinsic axial ratios of a triaxial galaxy. The method can be applied to bulges of spiral galaxies and to ellipticals containing sufficiently extended and kinematically regular gas disks. Required observational inputs are multi-colour surface photometry and the gas velocity field.

Motivation: It is now believed that giant elliptical galaxies are often triaxial systems (e.g. Binney 1985). There is increasing evidence that, despite their fast rotation, the bulges of some spiral galaxies are also triaxial (Zaritsky \& Lo 1986; M31: Lindblad 1956; the Galaxy: Gerhard \& Vietri 1986; barred galaxies: Kormendy 1982). Because of projection effects, the axial ratios of a triaxial galaxy are not readily determined and most attempts have therefore been statistical. Here we describe how in favourable circumstances the axial ratios of an individual triaxial system can be obtained directly from observations.

Input and assumptions: If applied to a triaxial bulge, our method requires photometry of the bulge, in several colours to remove the effects of dust, and the velocity field of the gas in the disk plane, at distances such that the potential is dominated by the bulge. Then, if we assume that the minor axis of the bulge and the spin axis of the disk are the same, that the disk is not flattened in its major plane, and that the bulge is non-rotating, and if the gas is in regular motion, the axis ratios of the bulge follow. In the case of an elliptical galaxy, the method requires the presence of a regular gas disk in one of the principal planes, with some gas inside $r_{e}$ and some gas well outside $r_{e}$, and a regular velocity field. Examples may be NGC 1097 (Davies, this conference) and Cen A (Bland, this conference).

The method: The axial ratios of a bulge in a disk galaxy can be obtained as a function of one (unknown) parameter, e.g. the true angle $\phi$ of the bulge intermediate axis wrt the disk apparent major axis (Stark 1977). The method uses as constraints $\theta$, the inclination angle of the disk, and $\psi$, the angle between the major axis of the isophotes of the bulge and disk $\left(=10^{\circ}\right.$ for M31). In an elliptical with extended gas disk, the outer parts replace the optical disk in the spiral to fix $\theta$ and $\psi$. - Here, we have computed gas velocity fields in various potentials, among which an illustrative de Vaucouleurs $(\mathrm{dV})$ model with $r_{e}=800 \mathrm{pc}$ and axial ratios $q=0.6$, assuming that the gas moves on closed orbits. Generally, the noncircular gas streamlines in the plane of the galaxy cause a tilted velocity field when 
projected onto the sky, in which the velocity contours cluster around the projected intermediate axis of the potential and significant minor axis rotation is present. Fig.1 shows, for the above $\mathrm{dV}$-model, one of the diagnostics that allow us to fix the remaining free parameter of the projection through the gas velocity field, and thus to obtain the intrinsic axial ratios of the triaxial body. In this case the velocities to be measured are of order $200 \mathrm{kms}^{-1}$ on the major axis, and large (up to $100 \mathrm{kms}^{-1}$ ) minor axis velocities occur. In an axisymmetric bulge, the rotation curve on the major axis determines the projected velocity anywhere. We can use this fact to test for a triaxial component in the potential, by plotting the velocity difference between the axisymmetric and the triaxial model, again as a funcion of position angle along the same ellipse as for Fig.1. Then one finds a typical cos $2 P A$ component, and this will tell us whether the application of Fig.1 to a given bulge is justified.

Conclusions: Regular gas velocity fields can be used to infer the existence of a triaxial component in the potential and to measure its intrinsic axial ratios. If the profile is de-Vaucouleurs-like, the effects are easily measurable for $q \leq 0.9$; more centrally concentrated models have more circular orbits; then flattenings of order $q \leq 0.7$ are measurable.

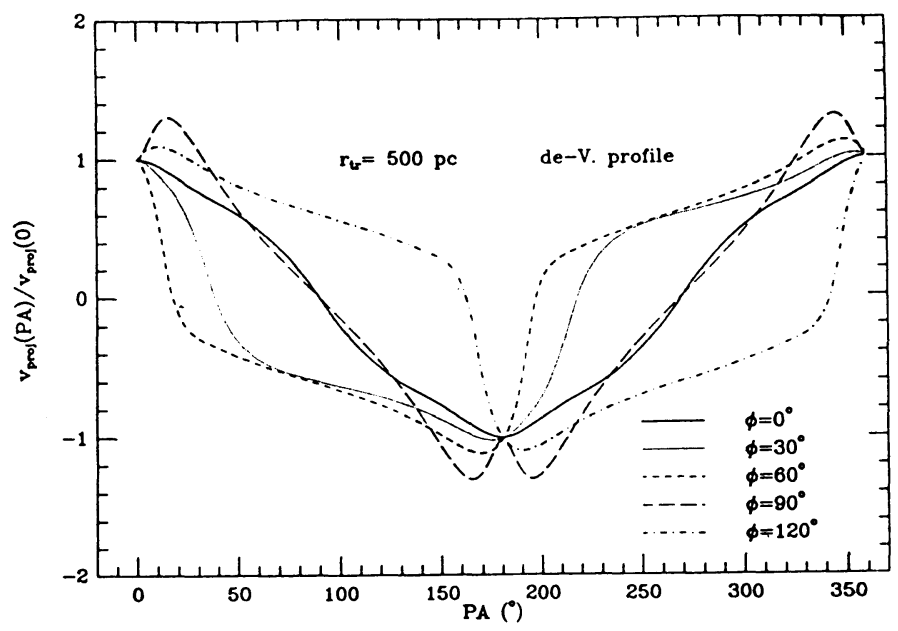

Fig.1: Gas velocity in the de Vaucouleurs model with $q=0.6$, projected along the line of sight, as a function of position angle along an ellipse on the plane of the sky that corresponds to a circle with $r=500 \mathrm{pc}$ in the plane of the galaxy, and for various choices of the unknown angle $\phi$. These velocities are normalized to the projected velocity measured on the disk major axis, so that these curves are independent of the choice of $\mathrm{M} / \mathrm{L}$.

\section{REFERENCES}

Binney, J.J., 1985. Mon. Not. R. astr. Soc. 212, 767.

Gerhard, O.E., Vietri, M., 1986. Mon. Not. R. astr. Soc., 223, 377.

Kormendy, J., 1982. Astrophys. J. 257, 75.

Lindblad, B., 1956. Stockholm Obs. Ann. 19, no.2.

Stark, A.A., 1977. Astrophys. J. $213,368$.

Zaritsky, D., Lo, K.Y., 1986. Astrophys. J. 303, 66. 